\section{Convulsiones febriles}

\author{
Febrile seizures
}

\section{DEFINICIÓN Y EPIDEMIOLOGÍA}

La Liga Internacional de Lucha contra la Epilepsia y la Organización Mundial de la Salud consideran a las convulsiones febriles como eventos comunes y benignos de la etapa infantil. Las convulsiones febriles son la forma más frecuente de crisis convulsiva en la infancia y afectan de 2 a $4 \%$ de los menores de 5 años en Estados Unidos y Europa, de 9 a 10\% en Japón y hasta a $14 \%$ en Guam. Son episodios convulsivos en el curso de una enfermedad febril en ausencia de epilepsia, desequilibrio hidrometabólico severo o neuroinfección. Ocurren en niños entre los 6 meses y los 5 años de edad con una incidencia máxima a los 18 meses. La temperatura asociada con el proceso infeccioso es frecuentemente mayor de $38.4^{\circ} \mathrm{C}$.

Las crisis convulsivas febriles son simples cuando los episodios son generalizados, únicos en 24 horas y con duración menor de 5 minutos; se denominan complejas cuando tienen un inicio focal, son múltiples en un día o duran más de 10 minutos. Alrededor del $65 \%$ de los pacientes presenta convulsiones febriles simples; $30 \%$ tienen al menos un evento parcial complejo y $5 \%$ desarrolla estado convulsivo de tipo febril. El $57 \%$ de las convulsiones febriles se presenta en las primeras 24 horas del cuadro febril, $22 \%$ en la primera hora de inicio de la fiebre $y$ $21 \%$ después del primer día de enfermedad.

\section{FACTORES DE RIESGO}

Se han encontrado algunos factores que incrementan hasta en $30 \%$ el riesgo de desarrollar convulsiones febriles:

- tener un familiar (de primer orden) con antecedente de convulsiones febriles

- estancia en cunero mayor de 30 días
Matilde Ruiz-García

Jefa del Servicio de Neurología Instituto Nacional de Pediatría

Recibido: 2 de septiembre del 2015

Aceptado: 10 de septiembre del 2015

Correspondencia: Matilde Ruiz García Servicio de Neurología Instituto Nacional de Pediatría Insurgentes Sur 3700- C CP 04530 México, D.F. matilderuizg@gmail.com

Este artículo debe citarse como Ruiz-García M. Convulsiones febriles. Acta Pediatr Mex 2015;36:424-427. 
- retraso psicomotor

- asistencia a una guardería

Aunque la mayoría de las crisis convulsivas febriles son únicas y aisladas $30 \%$ de los niños experimentará un segundo evento y aproximadamente $10 \%$ desarrollará tres o más. Los factores de asociados con recurrencia son:

- historia familiar de convulsiones febriles

- edad menor de 18 meses

- incremento rápido y elevación máxima de temperatura

- duración de la fiebre

El desarrollo psicomotor anormal, las crisis convulsivas parciales, el género y el origen étnico no se asocian con riesgo incrementado de recurrencia de convulsiones febriles.

\section{Epilepsia}

Entre 2 y $10 \%$ de los pacientes con convulsiones febriles desarrollará epilepsia en etapas posteriores. Los pacientes con antecedentes familiares de epilepsia frecuentemente presentan desarrollo psicomotor anormal y crisis convulsivas febriles complejas y repetitivas.

- La epilepsia puede ser parcial generalizada y es importante recordar que las convulsiones febriles pueden ser la primera manifestación clínica de síndromes epilépticos como la epilepsia mioclónica severa de la infancia, el síndrome de Dravet o el síndrome de Doose.

Todavía es controvertido si las convulsiones febriles representan un factor específico de susceptibilidad en edad para el desarrollo de epilepsia en un futuro y si existe una verdadera relación causal entre convulsiones febriles prolongadas y epilepsia, especialmente la del lóbulo temporal, o si los pacientes son portadores de un trastorno microscópico de la migración.

\section{Retraso mental y mortalidad}

No se ha demostrado que los pacientes con convulsiones febriles tengan mayores morbilidad y mortalidad ni que las condiciones cognoscitivas de estos pacientes sean diferentes a las de la población general.

\section{Genética}

Existe un patrón multifactorial de herencia en las convulsiones febriles; sin embargo, en algunas familias existe un patrón autosómico dominante relacionado con los cromosomas 18 y 19.

\section{EVALUACIÓN INICIAL}

Ante el primer episodio de convulsiones febriles es necesario realizar una evaluación que permita descartar meningitis, encefalitis, desequilibrio hidroelectrolítico severo y otras enfermedades agudas graves que ponen en riesgo la vida de los pacientes. Es indispensable obtener una historia clínica cuidadosa y una exploración física completa que permitan establecer si el paciente requiere estudios de extensión y si es necesario realizar una punción lumbar, ya que la incidencia de la meningitis en niños con convulsiones febriles es únicamente de entre 2 y $5 \%$.

La Academia Americana de Pediatría recomienda realizar punción lumbar en pacientes menores de 12 meses con convulsiones febriles y sugiere una evaluación cuidadosa de los pacientes entre 12 y 18 meses de edad. Pero la considera innecesaria en mayores de 18 meses cuando no tengan signos sospechosos de afectación cerebral.

Se han identificado cuatro situaciones de riesgo que deben considerarse cuando un paciente acude a emergencia por convulsiones febriles: 
- visita médica en las 48 horas previas con administración de antibióticos

- evento convulsivo a la llegada a emergencias

- crisis parciales complejas prolongadas

- examen físico neurológico anormal

No debe realizarse electroencefalograma durante las primaras horas poscrisis; se recomienda hacerlo 2 semanas después para evitar alteraciones inespecíficas y temporales secundarias al propio evento convulsivo.

\section{TRATAMIENTO}

El tratamiento recomendado dependerá de si el paciente acude en fase aguda a un centro de urgencias o si es referido al consultorio para evaluación de recurrencia de convulsiones febriles (Figuras 1 y 2 2). Si el paciente acude a urgencias por un evento convulsivo horas o minutos antes, y se encuentra reactivo, deberá hacerse la historia clínica y realizar la evaluación integral para decidir si son necesarios estudios de extensión o únicamente control térmico y vigilancia.

Si a su llegada a emergencias se encuentra convulsionando deberá controlarse la temperatura e indicarse diazepam por vía rectal para yugular el evento convulsivo; cuando las convulsiones febriles no cedan con el manejo benzodiacepínico apropiado deberá iniciarse tratamiento de estado epiléptico. La mayoría de las convulsiones febriles son breves y autolimitadas; suceden generalmente en casa y

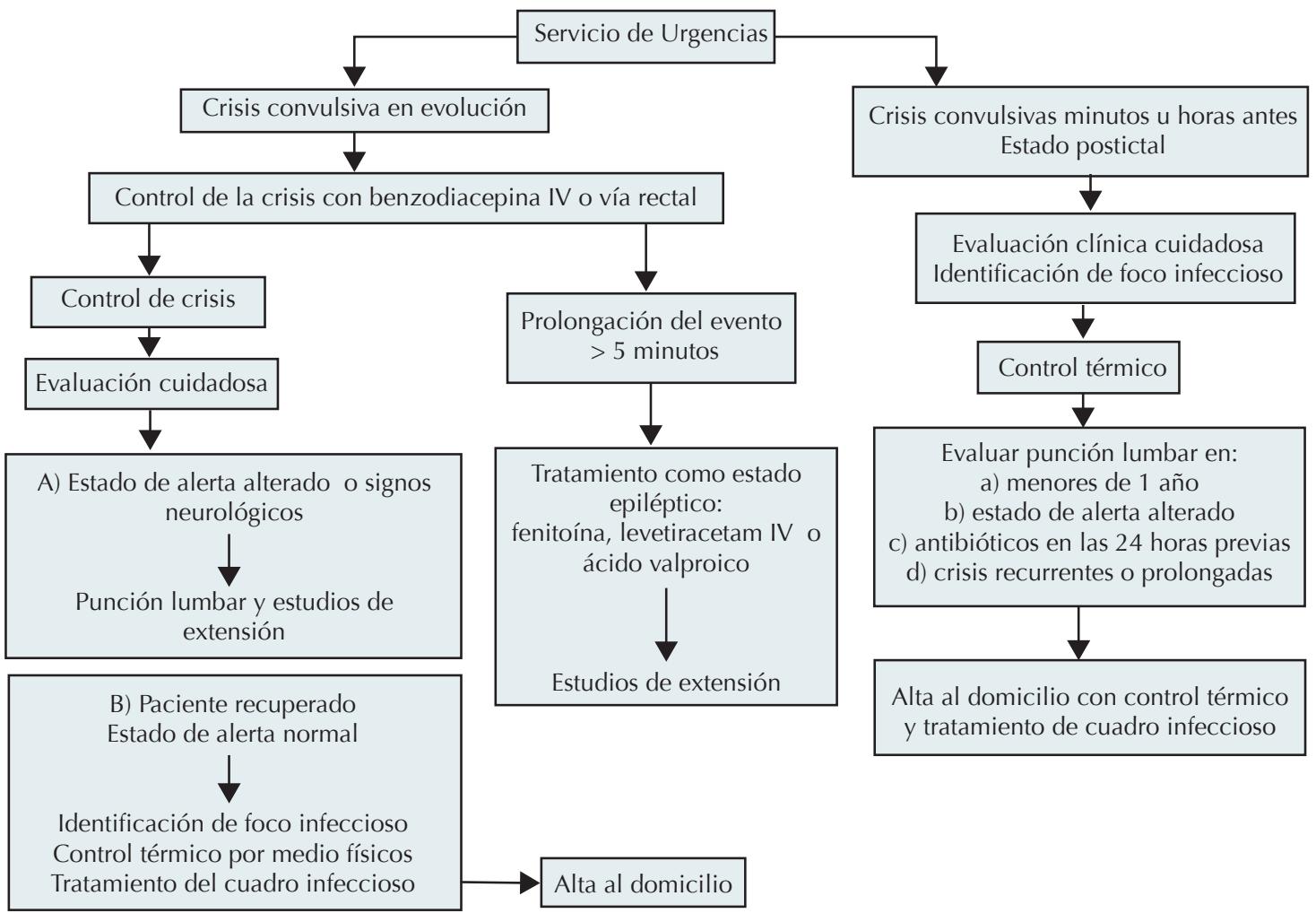

Figura 1. Tratamiento de las convulsiones febriles en la fase aguda. 


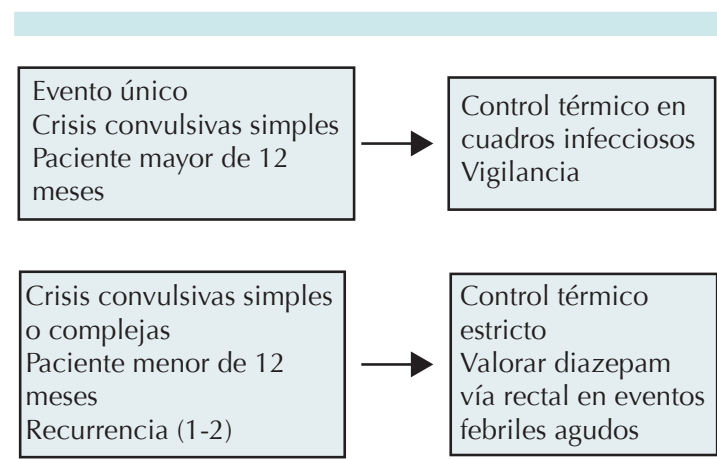

\begin{tabular}{|l|l|}
$\begin{array}{l}\text { Crisis convulsivas } \\
\text { complejas } \\
\text { Paciente menor de } 18 \\
\text { meses } \\
\text { Más de } 3 \text { recurrencias }\end{array} \rightarrow \begin{array}{l}\text { Control térmico estricto } \\
\text { Diazepam vía rectal en } \\
\text { eventos infecciosos } \\
\text { Valorar ácido valproico } \\
\text { o levetiracetam en } \\
\text { terapia crónica }\end{array}$ \\
\hline
\end{tabular}

Figura 2. Seguimiento de las convulsiones febriles.

es por ello que debe recomendarse control térmico estricto o utilización de diazepam rectal para evitar la recurrencia en pacientes de riesgo.

\section{Tratamiento intermitente o de fase aguda}

\section{Antipiréticos}

El control térmico por medios físicos y con antipiréticos es el tratamiento racional y óptimo para evitar la presencia o la recurrencia de las convulsiones febriles. En cerca de $25 \%$ de los pacientes las convulsiones aparecen justo al inicio del evento febril y por lo tanto el control térmico puede ser insuficiente. Los antiinflamatorios no esteroides son los medicamentos de elección para el control de la fiebre y de los procesos inflamatorios agudos en la infancia, inhiben la ciclooxigenasa y reducen la síntesis de prostaglandinas en el organismo y en el cerebro. Son seguros y eficaces cuando se manejan en dosis terapéuticas, aunque pueden producir efectos colaterales como hiporexia, vómito, gastritis.

\section{Benzodiacepinas}

La administración de diazepam por vías oral o rectal, al inicio de los episodios febriles en los lactantes y en los preescolares, ha demostrado reducir estadísticamente el riesgo de recurrencia en pacientes con convulsiones febriles; sin embargo, se deberán considerar los efectos de sedación que presentará el paciente.

\section{Tratamiento crónico con antiepilépticos}

Cada vez es menos frecuente el tratamiento farmacológico continuo con antiepilépticos a pacientes con convulsiones febriles. La indicación se limita a quienes han presentado 3 o más eventos, lo que representa únicamente $9 \%$ de los casos. Es importante mencionar que los estudios epidemiológicos no han podido demostrar que los pacientes con convulsiones febriles y tratamiento antiepiléptico crónico tengan menos riesgo de desarrollar epilepsia que los que no reciben tratamiento farmacológico. Los fármacos eficaces son los barbitúricos y el ácido valproico.

\section{LECTURAS RECOMENDADAS}

1. Summary of recommendations for the management of infantile seizures: Task Force Report for the ILAE Commission of Pediatrics. Wilmshurst JM, Gaillard WD, Vinayan KP, Tsuchida TN, Plouin P, Van Bogaert P, Carrizosa J, Elia M, Craiu D, Jovic NJ, Nordli D, Hirtz D, Wong V, Glauser T, Mizrahi EM, Cross JH. Epilepsia 2015;56(8):1185-97. doi: 10.1111/epi.13057

2. American Academy of Pediatrics Steering Committee on Quality Improvement and Management, Subcomittee on Febrile Seizures. Febriles seizures: clinical practice guideline for the long-term management of the child with simple febriles seizures. Pediatrics 2008;121(6):1281-1286.

3. Graves R, Oehler K, Tingle L. Febrile seizures: Risks, Evaluation, and prognosis. American Family Physician 2012;85(2):149-153.

4. Grill M, Yu-Tze Ng. Simple febrile seizures plus (SFS +): More than one febrile seizures within 24 hours is usually okay. Epilepsy and behavior 2013;(27):472-476.

5. Sfaihi L, Maaoloul I, Kmiha S, Aloulou H, Chabchoub I, et al. Febrile seizures: an epidemiological and outcome study of 482 cases. Childs Nerv Syst 2012;28(10):1779-84. 